\title{
Case of Recurrent Paracoccidioidomycosis: 25 Years After Initial Treatment
}
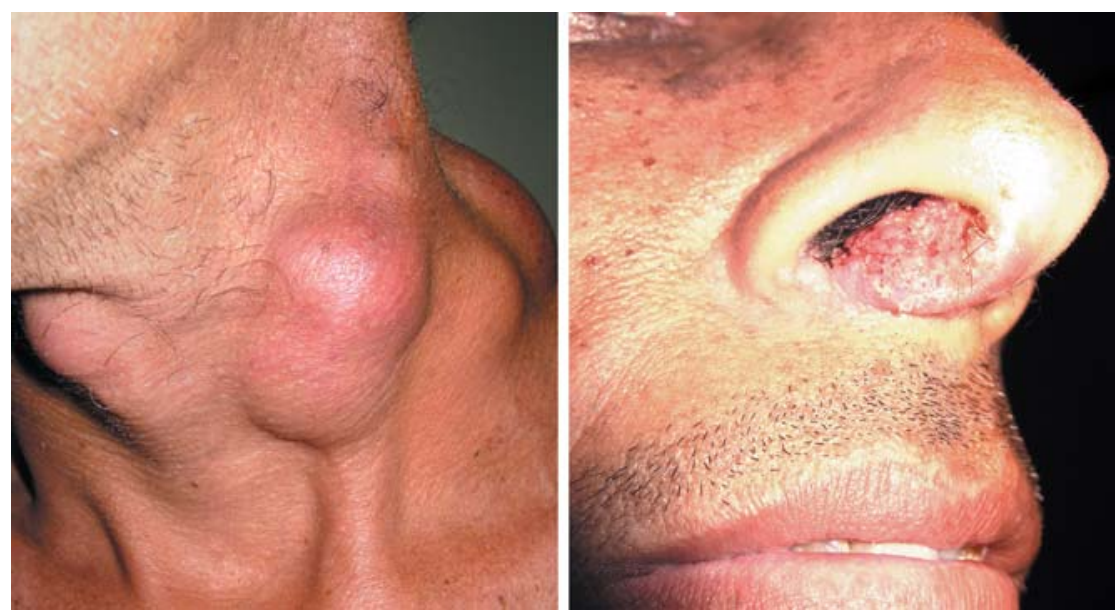

Figure 1. Cervical lymph node enlargement. Figure 2. Nasal mucosa lesion.

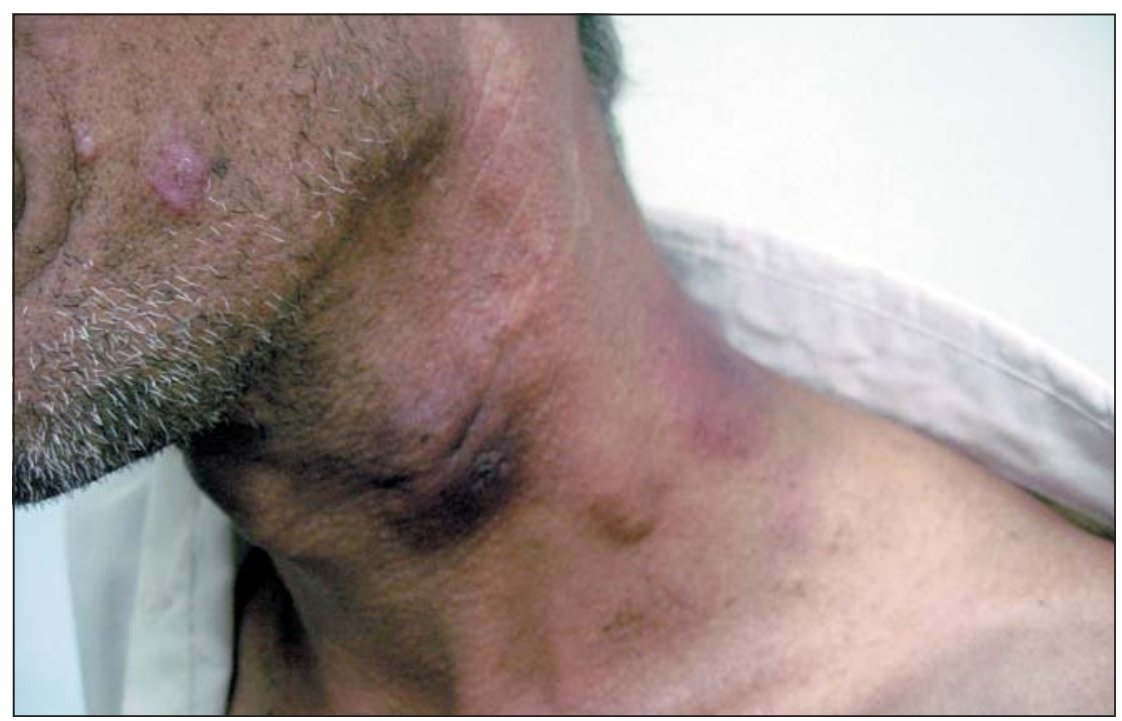

A43 year-old man, farmer, born in Santo Antônio do Norte, Minas Gerais, Brazil, developed Paracoccidioidomycosis (PCM) in 1983, when he was eighteen. He presented cervical, submandibular, supraclavicular, axillary and inguinal lymph node enlargement, and also involvement of the oral mucosa, lungs and guts. The diagnosis was made through the finding of Paracoccidioides brasiliensis in lymph node secretion and phlegm. He was admitted to the Hospital das Clinicas of the Universidade Federal de Minas Gerais and treated with Sulfadiazine, Sulfamethoxazole/ Trimethoprim and Ketoconazole for two months. After discharged from hospital he had no ambulatorial follow up or took any medicine. He revealed moderate tabagism and alcohol intake. In 1991 moved out to the metropolitan area of Belo Horizonte and became a doorman. He had no contact with any endemic area of PCM ever since. In 2004 he quitted tobacco smoking and alcohol drinking.

In January of 2008 he started loosing weight and showed dysphonia, odynophagia, vomiting, abdominal pain, low gastrointestinal hemorrhage, dyspnea, cough with expectoration and enlargement of cervical (Figure 1), axillary, navel and inguinal lymph node with suppuration. He also exhibited plaques and papules on the face and neck; ulcerated-vegetative lesions in the

Figure 3. Cervical lymph node and skin aspect after 40 days of treatment.

orofaringeal and nasal mucosa (Figure 2). Under suspicion of neoplasia it was indicated lymph node and orofaringeal biopsies, culture of lymph node secretion and sorology that were all positive for PCM. Complementary exams showed hemoglobin of $8 \mathrm{~g} / \mathrm{dL}$, hematocrit of 29\%, MVC of $68 \mathrm{fl}, \mathrm{MCH} 19 \mathrm{pg}$, ESR of $82 \mathrm{~mm}$ (1 hour), serum ferritin of $312 \mathrm{ng} / \mathrm{mL}$. Sorology for Chaga's Disease, HBV, HCV and HIV were negative and tuberculin test did not react. The Chest Xray, parasitologic exam of feces, abdominal ultra-sonography and upper gastrointestinal endoscopy were all normal. It was started Sulfametoxazol 800 mg and Trimetropim 160 mg every 12 hours. Forty days later the patient showed great recovery and improvement of cutaneus and lymph node lesions (Figure 3).

The Paracoccidioides brasiliensis may lay dormant in the organism and only express the disease several years after primo infection. This case report shows PCM reactivation 25 years after the first treatment that was considered "curative”. It indicates the huge latency (a quarter century) of the fungus. The patient did not show any predisposal factor associated with PCM (rural contact, tabagism or etilism). The disease presents a long time convivence and should be treated imediately after the first sign of recrudesence. The recurrence-relapse must be established with better precision and therefore periodical clinical control is fundamental. Recent studies based on chemokines analysis concluded that the minimal treatment should be of 36 months however it is necessary to establish clinical and laboratorial criteria to define a better cure control.

Stanley de Almeida Araújo ${ }^{1}$, Laura de Godoy Rousseff Prado², Juliana Marcia Ribeiro Veloso ${ }^{2}$ and Enio Roberto Pietra Pedroso ${ }^{1}$ ${ }^{1}$ Curso de Pós-Graduação em Ciências da Saúde: Infectologia e Medicina Tropical, Faculdade de Medicina, Universidade Federal de Minas Gerais; ${ }^{2}$ Acadêmicas do $11^{\circ}$ Período do Curso de Medicina. Faculdade de Medicina da Universidade Federal de Minas Gerais, Belo Horizonte, MG, Brazil. E-mail: stanleyaa@gmail.com 